\title{
Cardiac Rupture of the Junction of the Right Atrium and Superior Vena Cava in Blunt Thoracic Trauma
}

\author{
Chun Sung Byun, M.D., Il Hwan Park, M.D., Tae Hoon Kim, M.D. , Eunbi Lee, M.D. , \\ and Joong Hwan Oh, M.D., Ph.D. \\ Department of Thoracic and Cardiovascular Surgery, ${ }^{*}$ Department of Emergency Medicine, ${ }^{\dagger}$ Department of Anesthesiology and Pain Medicine, \\ Yonsei University Wonju College of Medicine, Wonju, Korea
}

\begin{abstract}
Cardiac rupture following blunt thoracic trauma is rarely encountered, since it commonly causes death at the scene. With advances in critical care, blunt cardiac rupture has been successfully treated with well-organized team approach including an emergency physician, anesthesiologist, and cardiac surgeon. We encountered a patient with blunt cardiac rupture of the junction of the superior vena cava and right atrium that extended $7 \mathrm{~cm}$ to the right ventricular junction. The patient was successfully resuscitated after a closed thoracostomy and pericardiocentesis with fluid loading. Cardiac injury was repaired via mid-sternotomy without cardiopulmonary bypass. The patient recovered without complications and was discharged on the 7 th day after surgery.
\end{abstract}

Key Words: heart injuries; heart rupture; pericardiocentesis; trauma, blunt injuries.

Cardiac trauma is common in blunt injuries of the anterior chest wall. However, survival after a cardiac rupture is rarely encountered clinically, as only a small percentage of patients reach the hospital alive and requiring treatment. Because of the severity of the disease itself, treatment requires a well-organized team including emergency medicine, anesthesiology and cardiovascular surgery from diagnosis to treatment to increase the survival rate after cardiac rupture. Previous reports have shown that the incidence of blunt traumatic cardiac rupture ranges from $0.16-2 \%$.[1,2] We report one case of successful treatment of a large cardiac rupture due to blunt trauma.

\section{Case Report}

A 41-year male patient came to our emergency center by wheel chair with blunt trauma to the anterior chest wall due to direct compression from a factory machine. The patient appeared acutely ill, with a pulse rate of 118 beats per minute, a blood pressure of $83 / 41 \mathrm{mmHg}$, a normal range of respiratory rate, and alert mental status during admission. The extended focused

Received on October, 20, 2014 Revised on November 21, 2014 Accepted on November 24, 2014

Correspondence to: Joong Hwan Oh, Department of Thoracic and Cardiovascular Surgery, Yonsei University Wonju College of Medicine, Wonju Severance Christian Hospital, 20 Ilsan-ro, Wonju 220-701, Korea

Tel: +82-33-742-1321, Fax: +82-33-741-0666

E-mail: mdjhoh@yonsei.ac.kr

*No potential conflict of interest relevant to this article was reported. assessment with sonography for trauma (eFAST) showed a right pneumothorax and cardiac tamponade resulting from a large hematoma in the pericardium (Fig. 1). Initial management included a closed thoracostomy, normal saline infusion, and transfusion of 2 units of packed red blood cells. Chest radiography was almost normal, except for a slightly enlarged right heart (Fig. 2). There was no injury to the ribs, sternum, 


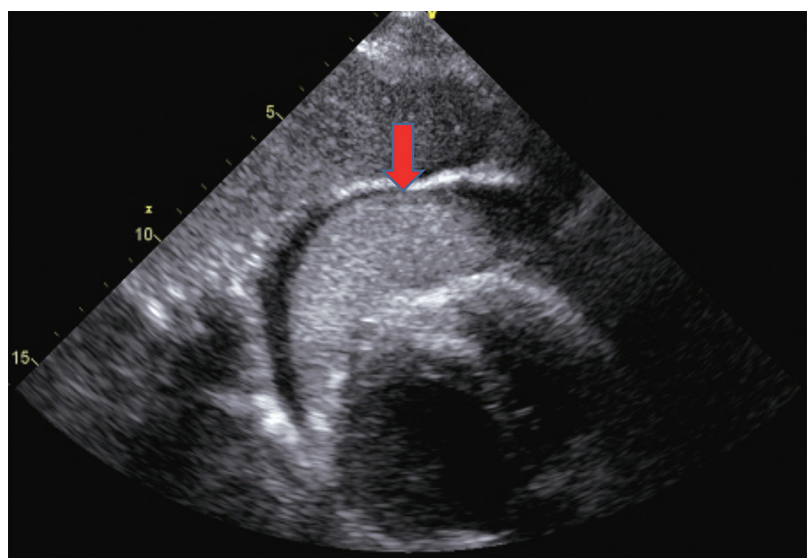

Fig. 1. The eFAST showed cardiac tamponade resulting from a large hematoma in the pericardium (red arrow). eFAST: extended focused assessment with sonography for trauma.

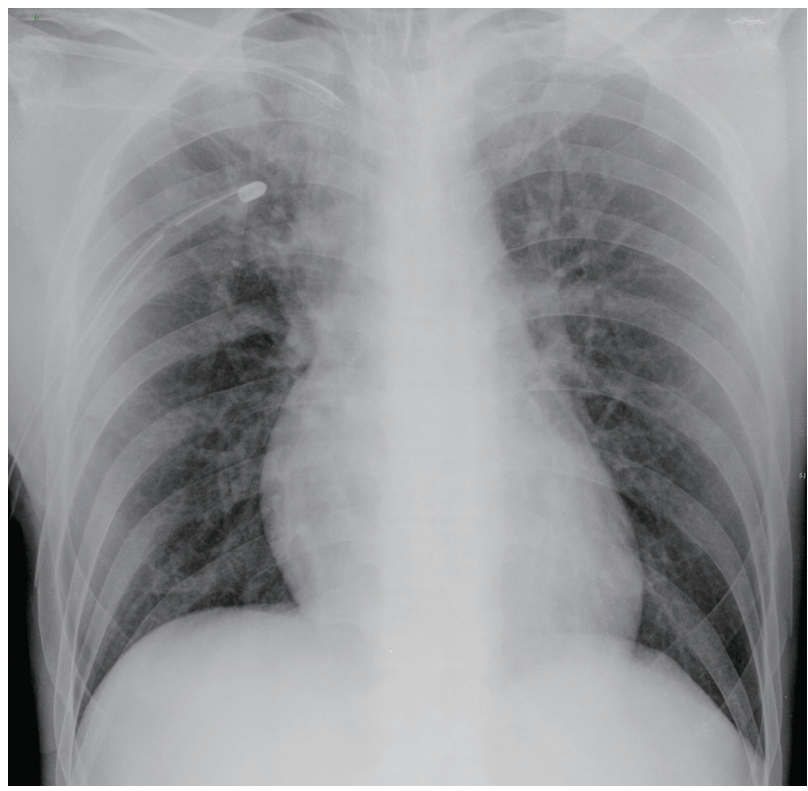

Fig. 2. Chest radiography was almost normal, except for the slightly enlarged right heart. The right chest tube was used to treat traumatic pneumothorax.

or abdominopelvic area after physical examination. Laboratory data showed mildly decreased hemoglobin at $12.5 \mathrm{~g} /$ $\mathrm{dL}$, increased troponin I at $0.333 \mathrm{ng} / \mathrm{mL}$ (normal $<0.046$ $\mathrm{ng} / \mathrm{mL}$ ), and compensated metabolic acidosis with a base excess of $-10.8 \mathrm{mmol} / \mathrm{L}$ in the blood. The emergency physician performed subxiphoid drainage of pericardial blood in emergency room under echocardiography (Fig. 3). The subxiphoid drainage relieved cardiac tamponade within minutes to earn time for adequate preparation for the emergent operation.

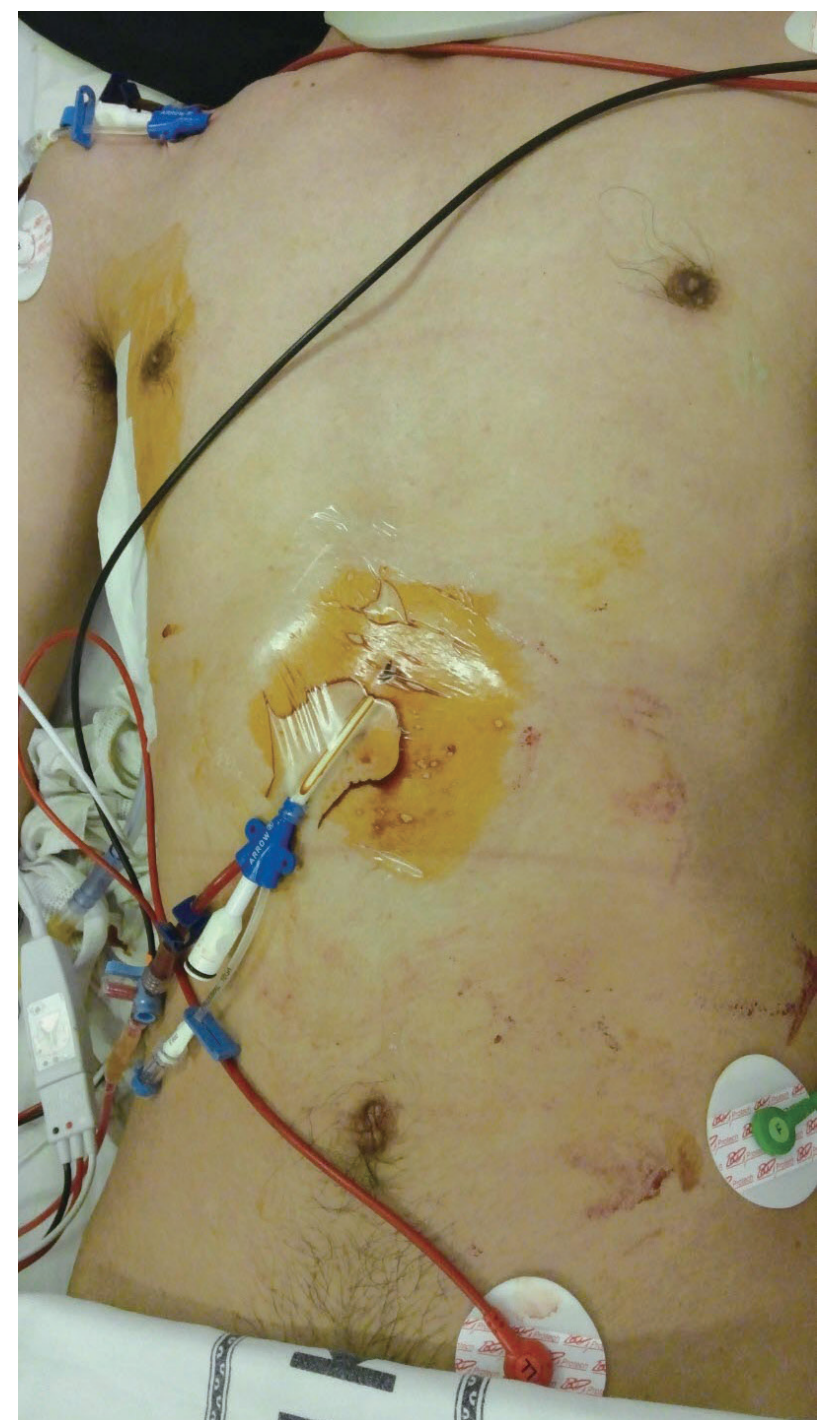

Fig. 3. Initial resuscitation with subxiphoid pericardial drainage was performed in emergency room under echocardiography.

The surgery was performed via mid-sternotomy. Fresh dark blood gushed out after pericardial incision. The cardiac injury site was compressed by fingers while blood was suctioned out. The injury was a superior vena cava (SVC) and right atrium (RA) junctional rupture with a deep laceration about $7 \mathrm{~cm}$ into the right ventricular junction (Fig. 4). The deep laceration was clamped under a Satinsky vascular clamp placed at the base of the RA appendage and another vascular clamp to the distal SVC. After clamping, gushing blood was decreased, and the injury site was roughly repaired with $3 / 0$ polypropylene suture over 3 minutes. After the clamp was taken off, the injury site was compressed with fingers. Because the heart was so shrunken with visu- 


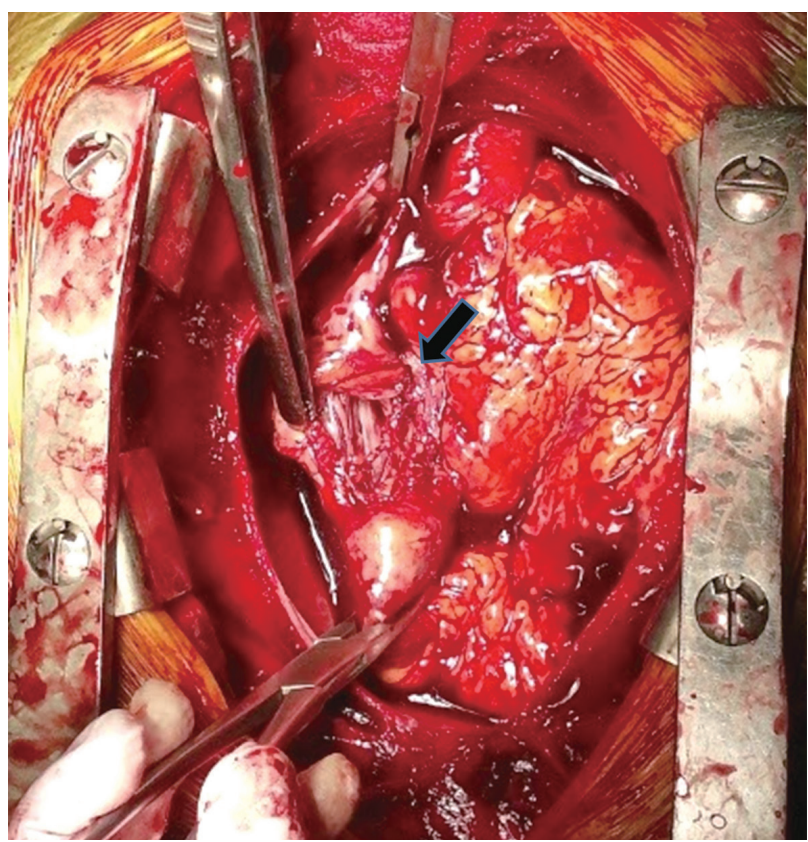

Fig. 4. The cardiac injury included rupture of the junction of the superior vena cava and the right atrium, with a deep $7 \mathrm{~cm}$ laceration into right ventricular junction (black arrow).

ally poor left ventricular contraction after clamping, definite fine suturing may need long clamping times, which may lead to poor neurologic outcomes. During the surgery, anesthesiologists supported heart function with massive volume infusion and transfusion via the femoral vein while clamping. The patient's vital signs improved during fluid resuscitation. During the second clamping of the SVC and RA, the heart was definitively repaired with $4 / 0$ polypropylene suture with Teflon pledget reinforcement over 5 minutes. The heart returned to normal contractility. The postoperative course was uneventful and the patient was discharged seven days after surgery without sequelae.

\section{Discussion}

Patients with cardiac rupture after blunt thoracic trauma hardly survived, and most of them die at the accident place or in the emergency room before the heart injury is revealed.[37] Severe precordial impaction with heart compressing between the sternum and spine is one possible theory. Another popular mechanism is rapid deceleration with disrupting the connection of the both atria from the vena cava and pulmo- nary veins.

The most common scenario of blunt traumatic cardiac rupture is cardiac tamponade. It is because that most of bleeding ceased for a short time spontaneously by direct compression of hematoma in pericardium. The identification of cardiac tamponade may be delayed or neglected because of multiple coexisting injuries, inadequate hypovolemic correction, or due to lack of skill in sonography. Beck's triad (muffled heart sounds, engorged jugular vein and hypotension) lacks sensitivity and specificity in cases of multiple systemic traumas. Hypotension in a patient with blunt thoracic trauma who has no evidence of massive haemothorax, tension pneumothorax may alert surgeons to the possibility of blunt heart injury. The eFAST is a confined examination protocol, but it is a quick and safe diagnostic tool for unstable vital sign patient who can not transfer to a computed tomography room. In this case, an emergency physician performed eFAST and successfully detected the cardiac tamponade early.

Some centers do not prefer subxiphoid drainage in cases of unclear blunt traumatic cardiac rupture, but we did a subxiphoid pericardial drainage under echocardiography to improve the patient's vital signs with fluid resuscitation. Subxiphoid drainage requires less equipment and surgical technique. This was clamped shortly after blood pressure recovered. During this procedure, the anesthesiologist, cardiac surgeon, and operation room nurses prepared the operation promptly.

Another manifestation of blunt traumatic cardiac rupture is massive haemothorax from pericardial bleeding. This phenomenon occurs in approximately $10 \%$ of blunt cardiac injury patients who have pericardial tearing. $[8,9]$ Most noncardiac surgeons tend to do exploratory thoracotomy rather than median sternotomy in massive haemothorax. However, thoracotomy had limited view of the heart and ascending aorta, which may have contained critical injury. Extend a previous subxiphoid drainage, and is suitable for cardiopulmonary bypass or extracorporeal cardiopulmonary life support. When heart injury is identified under exploratory thoracotomy in a case of massive haemothorax, immediate change to median sternotomy should be performed without hesitation. For patients with a large haemothorax (particularly a right haemothorax), median sternotomy must be considered as the first exploratory incision for possible heart 
injury with pericardial tearing. Most right atrial and right ventricular bleeding can be repressed by direct finger compression or using direct vascular clamp, followed by sutures with pledget reinforcement.[10,11] During these procedures in the operation field, life support from the anesthesiologist including volume resuscitation, adequate transfusion and proper infusion of cardiac drugs is important for successful recovery.

In summary, patients with blunt traumatic cardiac rupture will last to be a difficult critical traumatic care. High suspicion, effective diagnostic protocols, proper surgical management and a well- organized team approach are essential for better outcomes.

\section{ORCID}

Chun Sung Byun http://orcid.org/0000-0001-5409-6798

\section{References}

1) Martin TD, Flynn TC, Rowlands BJ, Ward RE, Fischer RP: Blunt cardiac rupture. J Trauma 1984; 24: 287-90.

2) Perchinsky MJ, Long WB, Hill JG: Blunt cardiac rupture. The Emanuel Trauma Center experience. Arch Surg 1995; 130: 852-6.
3) Baillot R, Dontigny L, Verdant A, Vaillancourt R, Pagé A, Pagé P, et al: Intrapericardial trauma: surgical experience. J Trauma 1989; 29: 736-40.

4) Brathwaite CE, Rodriguez A, Turney SZ, Dunham CM, Cowley R: Blunt traumatic cardiac rupture. A 5-year experience. Ann Surg 1990; 212: 701-4.

5) Kato K, Kushimoto S, Mashiko K, Henmi H, Yamamoto Y, Otsuka T: Blunt traumatic rupture of the heart: an experience in Tokyo. J Trauma 1994;36:859-63.

6) Malangoni MA, McHenry CR, Jacobs DG: Outcome of serious blunt cardiac injury. Surgery 1994; 116: 628-32.

7) Shorr RM, Crittenden M, Indeck M, Hartunian SL, Rodriguez A: Blunt thoracic trauma. Analysis of 515 patients. Ann Surg 1987; 206: 200-5.

8) Elie MC: Blunt cardiac injury. Mt Sinai J Med 2006; 73: $542-52$.

9) Fukuoka M, Takeuchi T, Tsubota H, Mikuriya Y, Oda M, Murata S: Traumatic pericardial rupture involved with complication by blunt chest trauma. Jpn J Thorac Cardiovasc Surg 2004; 52: 423-5.

10) Hoffman L, Pierce D, Puumala S: Clinical predictors of injuries not identified by focused abdominal sonogram for trauma (FAST) examinations. J Emerg Med 2009; 36: 271-9.

11) Perchinsky MJ, Long WB, Hill JG: Blunt cardiac rupture. The Emanuel Trauma Center experience. Arch Surg 1995; 130: 852-6. 